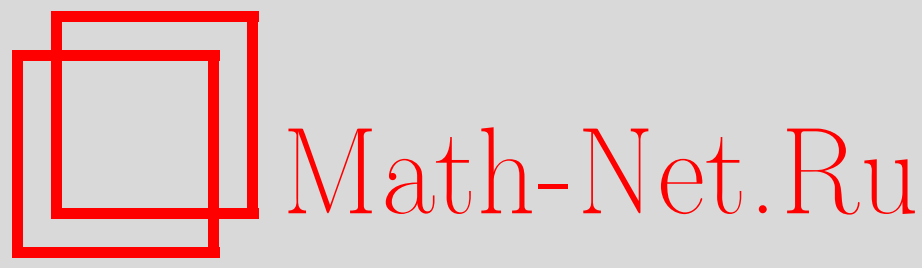

Д. И. Борисов, Об отсутствии лакун в нижней части спектра лапласиана с частым чередованием краевых условий в полосе, ТМФ, 2018, том 195, номер 2, 225-239

DOI: https://doi.org/10.4213/tmf9411

Использование Общероссийского математического портала Math-Net.Ru подразумевает, что вы прочитали и согласны с пользовательским соглашением http://www.mathnet.ru/rus/agreement

Параметры загрузки:

IP: 3.89 .185 .249

26 апреля 2023 г., 17:06:22

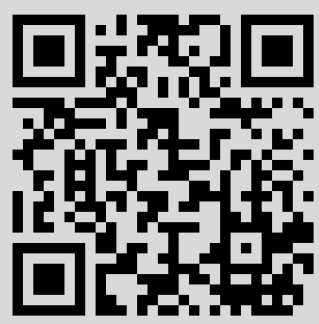


Том 195, № 2

май, 2018

(C) 2018 г. Д. И. Борисов ${ }^{* \dagger \ddagger}$

\title{
ОБ ОТСУТСТВИИ ЛАКУН \\ В НИЖНЕЙ ЧАСТИ СПЕКТРА ЛАПЛАСИАНА \\ С ЧАСТЫМ ЧЕРЕДОВАНИЕМ КРАЕВЫХ УСЛОВИЙ В ПОЛОСЕ
}

\begin{abstract}
Рассматривается лапласиан в плоской бесконечной прямой полосе с периодическим чередованием краевых условий. Показано, что при достаточно малом периоде чередования в нижней части спектра отсутствуют внутренние лакуны. В явном виде в терминах конкретных чисел и функций выписаны оценка сверху для величины периода и выражение для длины нижней части спектра, в которой гарантированно отсутствуют лакуны.
\end{abstract}

Ключевые слова: гипотеза Бете-Зоммерфельда, лакуна, периодический оператор, чередование краевых условий, лапласиан, бесконечная полоса.

DOI: https://doi.org/10.4213/tmf9411

\section{1. ВВЕДЕНИЕ}

Суть широко известной гипотезы Бете-Зоммерфельда состоит в том, что для достаточно широкого класса периодических дифференциальных операторов их существенный спектр содержит конечное число внутренних лакун. Такая гипотеза действительно нашла свое подтверждение для ряда операторов. Насколько нам известно, она была доказана для лапласиана и полигармонического оператора с младшими членами в многомерных пространствах и для операторов, сводящихся к ним заменами переменных. В работах [1]-[6] гипотеза Бете-Зоммерфельда была доказана для классического оператора Шредингера с периодическим потенциалом в пространствах различных размерностей. Случай магнитного оператора Шредингера

Исследование выполнено за счет гранта Российского научного фонда (проект № 17-11-01004).

* Институт математики с вычислительным центром Уфимского научного центра Российской академии наук, Уфа, Россия. E-mail: borisovdi@yandex.ru

${ }^{\dagger}$ Башкирский государственный педагогический университет им. М. Акмуллы, Уфа, Россия

${ }^{\ddagger}$ University of Hradec Králové, Hradec Králové, Czech Republic 
был успешно исследован в статьях [7], [8]. Оператор более высокого порядка, а именно полигармонический оператор с различными возмущениями, изучался в [9]-[11]. В этих работах гипотеза была доказана для большого класса операторов в случае, когда возмущение - это псевдодифференциальный оператор меньшего порядка, удовлетворяющий определенным условиям.

Гипотезу Бете-Зоммерфельда можно сформулировать и для периодических операторов в областях типа полос, цилиндров, слоев. В одном частном случае - для лапласиана, возмущенного ограниченным периодическим симметрическим оператором, в плоской бесконечной прямой полосе с краевым условием Дирихле - данная гипотеза была доказана в работе [12] при дополнительном предположении, что ширина полосы достаточно велика. Однако нам не удалось найти иных публикаций, где эта гипотеза доказывалась бы для более сложных операторов в цилиндрических областях или областях типа слоев.

Если гипотеза Бете-Зоммерфельда выполнена для какого-либо периодического самосопряженного дифференциального оператора, который к тому же полуограничен снизу, то это означает существование такой точки на вещественной оси, правее которой существенный спектр данного оператора представляет собой луч. Данную ситуацию можно интерпретировать как отсутствие лакун в верхней части существенного спектра. Это естественным образом порождает вопрос о выделении случаев, когда лакуны отсутствуют в нижней части спектра. Одновременное выполнение гипотезы Бете-Зоммерфельда для таких операторов означает отсутствие у них внутренних лакун в существенном спектре.

Результат такого типа приведен в работе [6] (см. теорему 15.2), где рассматривался лапласиан во всем пространстве размерности не меньше трех. К нему добавлялся ограниченный симметрический оператор, периодический относительно рациональной решетки; ограниченность понималась в смысле нормы операторов в пространстве $L_{2}$. Было показано, что если норма возмущения достаточно мала, то существенный спектр такого оператора вовсе не имеет внутренних лакун. Аналогичный результат для прямоугольных решеток был получен в теореме 15.6, а схожий результат для старших размерностей при условии рациональности решетки доказан в теореме 16.2 в работе [6]. Отметим также, что, поскольку в цитированных теоремах оператор рассматривался во всем пространстве, с помощью простого растяжения переменных случай малого возмущающего оператора сводится к случаю конечного оператора, но на малой ячейке периодичности. Другими словами, при выполнении условий данных теорем операторы не будут иметь внутренних лакун и для достаточно малых ячееек периодичности, что фактически следует из оценок, приведенных в утверждении теорем (см., например, неравенство (16.4) в [6]).

В настоящей работе мы рассматриваем оператор Лапласа в плоской бесконечной прямой полосе. На верхней границе задается краевое условие Дирихле, а на нижней - периодическое чередование краевых условий. А именно, нижняя граница периодическим образом разбивается на конечные отрезки, на которых поочередно задаются краевые условия Дирихле и Неймана (см. рис. 1). Наш основной результат утверждает, что если длины этих отрезков достаточно малы, то в нижней части существенного спектра рассматриваемого оператора отсутствуют внутренние лакуны. Оценки сверху для длин, гарантирующие такой результат, выписаны в конкретном 


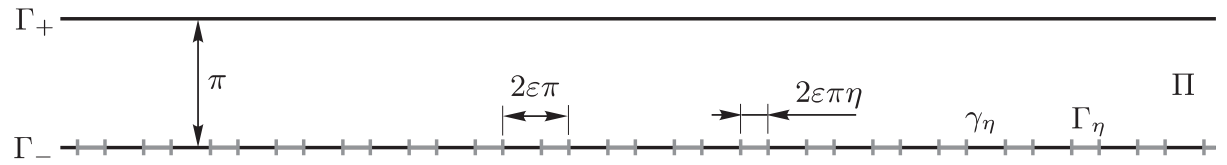

Рис. 1. Полоса с периодическим чередованием краевых условий.

числовом виде. Кроме того, в явном виде найдена длина нижней части спектра, для которой гарантировано отсутствие лакун.

Интерес к подобному исследованию спектральных лакун для модели с частой сменой краевых условий мотивирован серией работ [13]-[19] по операторному подходу к теории граничного усреднения. В этих работах все задачи рассматривались в плоских бесконечных полосах. Была доказана равномерная резольвентная сходимость операторов с возмущениями из теории граничного усреднения к соответствующим усредненным операторам и получены оценки скорости сходимости. Из этого результата стандартным образом вытекает сходимость спектров возмущенных операторов к спектрам усредненных операторов. Но даже в случаях, когда спектры усредненных операторов не содержали внутренних лакун, это еще не гарантировало отсутствие лакун в спектрах возмущенных операторов. Единственное, что можно было утверждать, - это убегание в бесконечность каждой имеющейся лакуны, т. е. увеличение расстояние от нуля до левого края лакуны. Скорость роста такого расстояния для задач с частой сменой краевых условий изучалась в статьях [15]-[17]. В частности, было показано, что расстояние от края спектра до первой лакуны по меньшей мере есть величина порядка $O\left(\varepsilon^{-2}\right)$, где $\varepsilon$ - малый параметр, описывающий длины отрезков с разными краевыми условиями.

Результат настоящей работы существенно лучше: мы показываем, что расстояние не меньше $O\left(\varepsilon^{-6}\right)$, причем оценка снизу для этого расстояния выписана в терминах малого параметра через конкретную функцию с конкретными числовыми константами.

В заключение отметим, что для модели периодического оператора, рассматриваемого в настоящей работе, естественно предположить выполнение гипотезы Бете-Зоммерфельда. Другими словами, мы предполагаем, что существенный спектр лапласиана с частой сменой краевых условий в полосе имеет лишь конечное число лакун, а в случае достаточно малой длины частей границы с различными краевыми условиями существенный спектр вовсе не должен иметь внутренних лакун. К сожалению, техника настоящей работы не позволила нам получить доказательство таких утверждений, и не удалось адаптировать известные нам методики из цитированных выше работ. Также не удалось пока реализовать в полной мере имеющиеся у автора идеи по доказательству данной гипотезы. Поэтому в настоящей работе мы ограничиваемся только доказательством отсутствия лакун в нижней части спектра.

\section{2. ПОСТАНОВКА ЗАДАЧИ И ОСНОВНОЙ РЕЗУЛЬТАТ}

Пусть $x=\left(x_{1}, x_{2}\right)$ - декартовы координаты в $\mathbb{R}^{2}, \Pi:=\left\{x: 0<x_{2}<\pi\right\}$ - горизонтальная полоса ширины $\pi, \varepsilon$ - достаточно малое положительное число.

В настоящей работе рассматривается лапласиан в полосе П с периодическим чередованием краевых условий, которое задается следующим образом. Нижняя граница 
$\Gamma_{-}:=\left\{x: x_{2}=0\right\}$ полосы П разбивается на отрезки (см. рис. 1$)$ :

$$
\begin{aligned}
\Gamma_{\eta}:=\bigcup_{j \in \mathbb{Z}}\left\{x:\left|x_{1}-\varepsilon \pi(2 j+1)\right|<\varepsilon \pi \eta\right\}, \\
\Gamma_{-}=\overline{\Gamma_{\eta}} \cup \overline{\gamma_{\eta}}, \quad \\
\gamma_{\eta}:=\bigcup_{j \in \mathbb{Z}}\left\{x:\left|x_{1}-2 \pi \varepsilon j\right|<\varepsilon \pi(1-\eta)\right\},
\end{aligned}
$$

где $\eta=\eta(\varepsilon)$ - дополнительный параметр, удовлетворяющий неравенству $0 \leqslant \eta \leqslant 1$. Никаких иных условий на зависимость $\eta$ от $\varepsilon$ не налагается.

Оператор вводится как лапласиан $\mathcal{H}_{\eta}:=-\Delta$ в П с краевым условием Дирихле на верхней границе $\Gamma_{+}:=\left\{x: x_{2}=\pi\right\}$ и в области $\gamma_{\eta}$ и с краевым условием Неймана в области $\Gamma_{\eta}$ (см. рис. 1). Математически строго оператор $\mathcal{H}_{\eta}$ мы определяем как полуограниченный самосопряженный оператор, соответствующий полуторалинейной форме в пространстве $L_{2}(\Pi)$ вида

$$
\mathfrak{h}_{\eta}(u, v):=(\nabla u, \nabla v)_{L_{2}(\Pi)}^{2} \quad \text { на } \quad \stackrel{\circ}{W}_{2}^{1}\left(\Pi, \Gamma_{+} \cup \gamma_{\eta}\right) .
$$

Здесь $\stackrel{\circ}{W}_{2}^{1}(\Omega, S)$ - пространство функций из $W_{2}^{1}(\Omega)$, заданных в некоторой области $\Omega$ и обращающихся в нуль на кривой $S$.

Основной объект нашего исследования - спектр оператора $\mathcal{H}_{\eta}$. Так как этот оператор периодичен, его спектр имеет зонную структуру и является объединением образов зонных функций. Зонные функции $E_{\eta}^{k}(\tau), k \geqslant 1,-$ это упорядоченные по возрастанию с учетом кратностей собственные значения соответствующих операторов на ячейке периодичности $\square:=\left\{x:\left|x_{1}\right|<\varepsilon \pi, 0<x_{2}<\pi\right\}$, зависящие от (масштабированного) квазиимпульса $\tau \in[-1 / 2,1 / 2)$. Соответствующий оператор на ячейке есть

$$
\mathcal{H}_{\eta}(\tau):=\left(i \frac{\partial}{\partial x_{1}}+\frac{\tau}{\varepsilon}\right)^{2}-\frac{\partial^{2}}{\partial x_{2}^{2}}, \quad x \in \square,
$$

с краевым условием Дирихле на верхней границе ячейки $\square$ и на $\partial \square \cap \gamma_{\eta}$, краевым условием Неймана на $\partial \square \cap \Gamma_{\eta}$ и периодическими краевыми условиями на боковых сторонах $l_{ \pm}$ячейки $\square, l_{ \pm}:=\left\{x: x_{1}= \pm \varepsilon \pi, x_{2} \in(0, \pi)\right\}$.

Оператор $\mathcal{H}_{\eta}(\tau)$ вновь вводим как оператор, соответствующий полуторалинейной форме в $L_{2}(\square)$ вида

$$
\left(\left(i \frac{\partial}{\partial x_{1}}+\tau\right) u,\left(i \frac{\partial}{\partial x_{1}}+\tau\right) v\right)_{L_{2}(\square)}^{2}+\left(\frac{\partial u}{\partial x_{2}}, \frac{\partial v}{\partial x_{2}}\right)_{L_{2}(\square)}^{2}
$$

заданной на $\stackrel{\circ}{W}_{2, \text { per }}^{1}\left(\square, \partial \square \cap\left(\Gamma_{+} \cup \gamma_{\eta}\right)\right)$. Здесь $\stackrel{\circ}{W}_{2, \text { per }}^{1}(\square, S)-$ пространство функций из $\stackrel{\circ}{W}_{2}^{1}(\square, S)$, удовлетворяющих периодическим краевым условиям на боковых границах $l_{ \pm}$.

Через $\sigma(\cdot)$ обозначим спектр оператора, через $[\cdot]$ - целую часть числа.

Основная цель работы - доказать отсутствие лакун в определенной части зонного спектра оператора $\mathcal{H}_{\eta}$. Основной результат сформулирован в следующей теореме.

TEOPEMA 1. Пусть

$$
\varepsilon \leqslant \varepsilon_{0}, \quad \varepsilon_{0}=\frac{\sqrt{2}}{160}-\frac{\sqrt{7}}{672}
$$


Положим

$$
K_{\varepsilon}:=\frac{25}{16} \frac{\varepsilon_{0}^{2}}{\varepsilon^{2}}+\frac{5}{16} \frac{\varepsilon_{0}}{\varepsilon^{2}} \sqrt{25 \varepsilon_{0}^{2}-16 \varepsilon^{2}}-\frac{1}{2} .
$$

Тогда часть спектра $\sigma\left(\mathcal{H}_{\eta}\right)$ оператора $\mathcal{H}_{\eta}$, лежащая на полуоси

$$
\left(-\infty, \frac{\left(\left[K_{\varepsilon}\right]+1\right)^{2}}{\varepsilon^{2}}\right],
$$

не содержит лакун.

Обсудим кратко основной результат. Утверждается, что при малом периоде чередования нижняя часть спектра свободна от внутренних лакун. При этом оценка сверху для допустимых значений $\varepsilon$ и длина нижней части, в которой гарантируется отсутствие лакун, выписаны явно. При уменьшении $\varepsilon$ длина нижней части спектра без лакун растет, по крайней мере как $O\left(\varepsilon^{-6}\right)$, что следует непосредственно из определения функции $K_{\varepsilon}$.

Тот факт, что на границе $\Gamma_{+}$задается именно первое краевое условие, не является существенным. Аналогичный результат будет верен и для краевого условия Неймана либо для третьего краевого условия с постоянным коэффициентом на $\Gamma_{+}$, но с другими константой $\varepsilon_{0}$ и функцией $K_{\varepsilon}$.

В заключение подчеркнем, что константа $\varepsilon_{0}$ в утверждении теоремы не является оптимальной, и ее можно улучшить, оставаясь в рамках техники нашей работы. Вместе с тем основная цель работы - продемонстрировать эффект отсутствия лакун в нижней части спектра, растущей с уменьшением $\varepsilon$. Поэтому в процессе вычислений мы сознательно делаем более грубыми несколько технических оценок с целью их упрощения, что в конечном счете и приводит к неоптимальной константе $\varepsilon_{0}$.

\section{3. СЧИТАЮЩИЕ ФУНКЦИИ}

В настоящем разделе мы обсуждаем ряд вспомогательных понятий и утверждений, которые далее будут необходимы для доказательства теоремы 1.

Для произвольного $L>0$ через $N_{\eta}(L, \tau)$ обозначим считающую функцию оператора $\mathcal{H}_{\eta}(\tau)$ - число собственных значений этого оператора с учетом кратностей, не превосходящих $L^{2} / \varepsilon^{2}$ :

$$
N_{\eta}(L, \tau):=\max \left\{k: E_{\eta}^{k}(\tau) \leqslant \frac{L^{2}}{\varepsilon^{2}}\right\} .
$$

Зонные функции $E_{\eta}^{k}(\tau)$ упорядочены по возрастанию:

$$
E_{\eta}^{1}(\tau) \leqslant E_{\eta}^{2}(\tau) \leqslant \cdots \leqslant E_{\eta}^{1}(\tau) \leqslant \cdots
$$

а потому при фиксированном $L$ число $\sup _{\tau \in[-1 / 2,1 / 2)} N_{\eta}(L, \tau)$ является количеством зонных функций, минимумы которых не превосходят $L^{2} / \varepsilon^{2}$, а аналогичная величина $\inf _{\tau \in[-1 / 2,1 / 2} N_{\eta}(L, \tau)$ дает число зонных функций, максимумы которых не превосходят $L^{2} / \varepsilon^{2}$. Далее для краткости вместо $\sup _{\tau \in[-1 / 2,1 / 2)}$ и $\inf _{\tau \in[-1 / 2,1 / 2)}$ мы пишем $\sup _{\tau}$ и $\inf _{\tau}$ и аналогично для максимумов и минимумов.

Перекрытие двух соседних зон спектра

$$
\left[\min _{\tau} E_{\eta}^{k}(\tau), \max _{\tau} E_{\eta}^{k}(\tau)\right] \quad \text { и } \quad\left[\min _{\tau} E_{\eta}^{k+1}(\tau), \max _{\tau} E_{\eta}^{k+1}(\tau)\right]
$$


эквивалентно выполнению неравенства

$$
\sup _{\tau} N_{\eta}(L, \tau)-\inf _{\tau} N_{\eta}(L, \tau) \geqslant 1
$$

при

$$
\min _{\tau} E_{\eta}^{k}(\tau) \leqslant \frac{L^{2}}{\varepsilon^{2}} \leqslant \max _{\tau} E_{\eta}^{k}(\tau) .
$$

В более общем виде это утверждение звучит так: зонный спектр оператора $\mathcal{H}_{\eta}$ между точками $\lambda_{-}$и $\lambda_{+}$не содержит спектральных лакун, если для всех значений $L^{2} / \varepsilon^{2} \in\left[\lambda_{-}, \lambda_{+}\right]$выполнено неравенство (3.2).

Основная идея доказательства теоремы 1 заключается в проверке неравенства (3.2) для всех возможных значений $L$. Главная трудность состоит в подходящей оценке левой части, так как задачи об определении вида функции $N_{\eta}(L, \tau)$ и тем более о нахождении ее глобальных минимума и максимума очень сложны. Поэтому наш следующий шаг - оценка левой части неравенства (3.2) через аналогичную разность, но для считающих функций более простых операторов.

В силу вилки Дирихле-Неймана оператор $\mathcal{H}_{\eta}(\tau)$ подчиняется оценке

$$
\mathcal{H}_{1}(\tau) \leqslant \mathcal{H}_{\eta}(\tau) \leqslant \mathcal{H}_{0}(\tau)
$$

в смысле квадратичных форм. Здесь $\mathcal{H}_{0}(\tau)$ - это оператор $\mathcal{H}_{\eta}(\tau)$ при $\gamma_{\eta}=\Gamma_{-}$, $\Gamma_{\eta}=\varnothing$. Оператор $\mathcal{H}_{1}(\tau)$ - это оператор $\mathcal{H}_{\eta}(\tau)$ при $\Gamma_{\eta}=\Gamma_{-}, \gamma_{\eta}=\varnothing$. Для зонных функций это дает неравенства

$$
E_{1}^{k}(\tau) \leqslant E_{\eta}^{k}(\tau) \leqslant E_{0}^{k}(\tau)
$$

Для соответствующих считающих функций $N_{0}, N_{1}$ отсюда получаем

$$
N_{0}(L, \tau) \leqslant N_{\eta}(L, \tau) \leqslant N_{1}(L, \tau)
$$

Следовательно, достаточным условием выполнения неравенства (3.2) является оценка

$$
\sup _{\tau} N_{0}(L, \tau)-\inf _{\tau} N_{1}(L, \tau) \geqslant 1
$$

С учетом целочисленности функций $N_{0}, N_{1}$ достаточно проверить более слабое неравенство

$$
\sup _{\tau} N_{0}(L, \tau)-\inf _{\tau} N_{1}(L, \tau)>0 .
$$

Выпишем теперь явную формулу для функций $N_{0}$ и $N_{1}$. Собственные значения оператора $\mathcal{H}_{0}(\tau)$ и соответствующие собственные функции легко находятся разделением переменных:

$$
\Lambda_{n, m}^{0}(\tau)=\frac{(n+\tau)^{2}}{\varepsilon^{2}}+m^{2}, \quad \Psi_{n, m}^{0}(x)=e^{i n x_{1} / \varepsilon} \sin m x_{2}, \quad n \in \mathbb{Z}, \quad m \in \mathbb{N} .
$$

Поэтому зонные функции $E_{0}^{k}(\tau)$ - это числа $\Lambda_{n, m}^{0}(\tau)$, упорядоченные по возрастанию с учетом кратностей. Принимая во внимание определение $(3.1)$ видим, что $N_{0}(L, \tau)$ есть число целых точек $(n, m)$ на плоскости, удовлетворяющих неравенству

$$
(n+\tau)^{2}+\varepsilon^{2} m^{2} \leqslant L^{2},
$$


а именно

$$
\begin{aligned}
N_{0}(L, \tau) & =\#\left\{(n, m):(n+\tau)^{2}+\varepsilon^{2} m^{2} \leqslant L^{2}, n \in \mathbb{Z}, m \in \mathbb{N}\right\}= \\
& =\sum_{n:|n+\tau| \leqslant L}\left[\frac{\sqrt{L^{2}-(n+\tau)^{2}}}{\varepsilon}\right]=\sum_{n=-[L+\tau]}^{[L-\tau]}\left[\frac{\sqrt{L^{2}-(n+\tau)^{2}}}{\varepsilon}\right] .
\end{aligned}
$$

Аналогично находим функцию $N_{1}(L, \tau)$. Соответствующие собственные значения и собственные функции имеют вид

$$
\begin{aligned}
& \Lambda_{n, m}^{1}(\tau)=\frac{(n+\tau)^{2}}{\varepsilon^{2}}+\left(m-\frac{1}{2}\right)^{2}, \\
& \Psi_{n, m}^{1}(x)=e^{i n x_{1} / \varepsilon} \cos \left(m-\frac{1}{2}\right) x_{2},
\end{aligned}
$$

Отсюда выводим формулу для $N_{1}(L, \tau)$ :

$$
\begin{aligned}
N_{1}(L, \tau) & =\#\left\{(n, m):(n+\tau)^{2}+\varepsilon^{2}\left(m-\frac{1}{2}\right)^{2} \leqslant L^{2}, n \in \mathbb{Z}, m \in \mathbb{N}\right\}= \\
& =\sum_{n:|n+\tau| \leqslant L}\left[\frac{\sqrt{L^{2}-(n+\tau)^{2}}}{\varepsilon}+\frac{1}{2}\right]= \\
& =\sum_{n=-[L+\tau]}^{[L-\tau]}\left[\frac{\sqrt{L^{2}-(n+\tau)^{2}}}{\varepsilon}+\frac{1}{2}\right] .
\end{aligned}
$$

\section{4. ДОКАЗАТЕЛЬСТВО ТЕОРЕМЫ 1}

Основная идея доказательства состоит в проверке неравенства (3.4) для всех возможных значений $L$. При этом рассматриваемые значения $L$ должны удовлетворять неравенству

$$
L^{2} \geqslant \varepsilon^{2} \inf \sigma\left(\mathcal{H}_{\eta}\right) .
$$

Из неравенства (3.3) с $k=1$ и равенств

$$
E_{1}^{0}(\tau)=\frac{\tau^{2}}{\varepsilon^{2}}+1, \quad E_{1}^{1}(\tau)=\frac{\tau^{2}}{\varepsilon^{2}}+\frac{1}{4}
$$

следует, что

$$
\frac{1}{4} \leqslant \inf \sigma\left(\mathcal{H}_{\eta}\right) \leqslant 1
$$

Поэтому параметр $L$ должен удовлетворять неравенству $L^{2} \geqslant \varepsilon^{2} / 4$.

При $L^{2}$, достаточно близких к $\varepsilon^{2} \inf \sigma\left(\mathcal{H}_{\eta}\right)$, обе функции $N_{0}(L, \tau)$ и $N_{1}(L, \tau)$ могут обращаться в нуль при некоторых $\tau$, и проверка неравенства (3.4) может быть затруднительной. Поэтому для таких значений $L$, а именно для $L \leqslant 1 / 2$, отсутствие лакун мы доказываем без использования неравенства (3.4), а уже при $L>1 / 2$ - на основе неравенства (3.4).

При проверке неравенства (3.4) достаточно сложно найти точно глобальные максимум и минимум функций $N_{0}$ и $N_{1}$ по $\tau$ при произвольном $L$, и решения этой 
задачи мы не знаем. Вместе с тем ясно, что для произвольных $\tau_{0}, \tau_{1} \in[-1 / 2,1 / 2]$ выполнено неравенство

$$
\sup _{\tau} N_{0}(L, \tau)-\inf _{\tau} N_{1}(L, \tau) \geqslant N_{0}\left(L, \tau_{0}\right)-N_{1}\left(L, \tau_{1}\right)
$$

Следующий шаг - это вопрос о подходящем выборе точек $\tau_{0}$ и $\tau_{1}$. Сразу отметим, что $N_{0}$ и $N_{1}$ - четные функции переменной $\tau$, поэтому можно предположить, что $\tau_{0}, \tau_{1} \in[0,1 / 2]$. Разумеется, наиболее оптимальный выбор - это точки глобального максимума функции $N_{0}$ и глобального минимума функции $N_{1}$. Вместе с тем неизвестно, как найти данные точки. Поэтому в качестве $\tau_{0}$ и $\tau_{1}$ предлагается взять такие точки, что $N_{0}\left(L, \tau_{0}\right)$ сравнительно мало отличается от $\sup _{\tau} N_{0}(L, \tau)$, а $N_{1}\left(L, \tau_{1}\right)$ сравнительно мало отличается от $\inf _{\tau} N_{1}(L, \tau)$. Нахождение таких точек аналитически - вновь весьма непростая задача. Однако ряд предварительных численных экспериментов подтолкнул нас к следующему наблюдению. В качестве $\tau_{0}$ следует выбирать точку $\tau=0$ или $\tau=1 / 2$, причем конкретный выбор зависит от дробной части величины $L$, которую дальше мы обозначаем как $\alpha:=\{L\}$. А именно, далее мы полагаем

$$
\tau_{0}=\left\{\begin{array}{lcl}
\frac{1}{2} & \text { при } & 0 \leqslant \alpha \leqslant \frac{1}{4} \\
0 & \text { при } & \frac{1}{4} \leqslant \alpha<\frac{3}{4} .
\end{array}\right.
$$

В качестве $\tau_{1}$ следует выбрать расстояние от $\alpha$ до ближайшего целого:

$$
\tau_{1}=\left\{\begin{array}{lll}
\alpha & \text { при } & 0 \leqslant \alpha<\frac{1}{2}, \\
1-\alpha & \text { при } & \frac{1}{2} \leqslant \alpha<1 .
\end{array}\right.
$$

У нас нет строгих аналитических аргументов в пользу указанного выбора точек $\tau_{0}, \tau_{1}$, но попробуем пояснить данный выбор на нестрогом уровне. Число $\tau_{0}$ следует выбирать так, что количество целых точек $(n, m)$, удовлетворяющих неравенству (3.6), было максимально большим из всех возможных. Из геометрических соображений сразу же можно предположить, что максимальное (или близкое к нему) количество таких точек будет при $\tau_{0}=0$ или $\tau_{0}=1 / 2$. Указанный конкретный выбор между $\tau_{0}=0$ и $\tau_{0}=1 / 2$ в зависимости от $\alpha$ был подсказан, с одной стороны, упомянутыми выше численными экспериментами; с другой стороны, мы немного ухудшили выбор по сравнению результатами численных экспериментов с целью некоторого упрощения ряда технических оценок, которые мы приводим ниже. Отметим также, что не для всех значений $\varepsilon$ и $L$ максимум функции $N_{0}(L, \tau)$ достигается при $\tau=0$ или $\tau=1 / 2$. Так, например, при $L=39.623, \varepsilon=0.0035$ мы получили

$$
N_{0}(L, 0)=704646, \quad N_{0}(L, 0.499088)=704816, \quad N_{0}(L, 0.5)=704808 \text {. }
$$

Выбор числа $\tau_{1}$ объяснить сложнее, и единственный аналитический аргумент здесь звучит следующим образом. При указанном выборе $\tau_{1}$ в формуле (3.7) для $N_{1}$ одно из слагаемых, соответствующих $n=-[L+\tau]$ или $n=[L-\tau]$, обращается в нуль. Видимо, это обстоятельство позволяет добиться достаточно малого значения $N_{1}$. 
Вновь отметим, что указанное значение $\tau_{1}$ не всегда совпадает с точкой минимума функции $N_{1}$. Так, при $L=39.623, \varepsilon=0.0035$ мы получили

$$
\begin{array}{ll}
N_{1}(L, 0.377)=704308, & N_{1}(L, 0.3758)=704303, \\
N_{1}(L, 0)=704679, & N_{1}(L, 0.5)=704856 .
\end{array}
$$

Далее в доказательстве мы рассматриваем пять случаев: случай $L \leqslant 1 / 2$ и четыре случая $L>1 / 2$ при $0 \leqslant \alpha \leqslant 1 / 4,1 / 4<\alpha<1 / 2,1 / 2 \leqslant \alpha<3 / 4$ и $3 / 4 \leqslant \alpha<1$.

4.1. Пересечение первых двух зон. Из формул (3.5) следует, что первые зонные функции $E_{i}^{2}(\tau)$ имеют вид

$$
E_{0}^{2}(\tau)=\min \left\{\frac{(1-\tau)^{2}}{\varepsilon^{2}}+1, \frac{\tau^{2}}{\varepsilon^{2}}+4\right\}, \quad E_{1}^{2}(\tau)=\min \left\{\frac{(1-\tau)^{2}}{\varepsilon^{2}}+\frac{1}{4}, \frac{\tau^{2}}{\varepsilon^{2}}+\frac{9}{4}\right\} .
$$

Отсюда и из (2.1), (4.1) вытекает, что

$$
\begin{gathered}
\max _{\tau} E_{1}^{1}(\tau)=\frac{1}{4 \varepsilon^{2}}+\frac{1}{4}, \quad \min _{\tau} E_{0}^{2}(\tau)=4, \\
\max _{\tau} E_{1}^{2}(\tau)=\frac{\left(1-2 \varepsilon^{2}\right)^{2}}{4 \varepsilon^{2}}+\frac{9}{4}=\frac{1+5 \varepsilon^{2}+4 \varepsilon^{4}}{4 \varepsilon^{2}} .
\end{gathered}
$$

Из полученных оценок и соотношений (2.1), (3.3) следует, что

$$
\max _{\tau} E_{\eta}^{1}(\tau) \geqslant \min _{\tau} E_{\eta}^{2}(\tau), \quad \max _{\tau} E_{\eta}^{2}(\tau) \geqslant \frac{1+5 \varepsilon^{2}+4 \varepsilon^{4}}{4 \varepsilon^{2}} .
$$

Таким образом, первые две зоны спектра перекрываются и часть спектра

$$
\left[\inf \sigma\left(\mathcal{H}_{\eta}\right), \frac{1+5 \varepsilon^{2}+4 \varepsilon^{4}}{4 \varepsilon^{2}}\right]
$$

не содержит спектральных лакун. Данному отрезку спектра соответствуют значения

$$
L^{2} \in\left[\varepsilon^{2} \inf \sigma\left(\mathcal{H}_{\eta}\right), \frac{1+5 \varepsilon^{2}+4 \varepsilon^{4}}{4}\right],
$$

поэтому далее достаточно рассмотреть случай $L^{2}>1 / 4$, т. е. $L>1 / 2$.

4.2. Вспомогательные оценки. Здесь мы считаем, что $L>1 / 2$, и приводим некоторые вспомогательные оценки, которые будут использоваться далее в доказательстве.

Введем обозначения

$$
\begin{gathered}
F_{0}(L, X, A):=2 F_{1}(L, X, 0)-F_{1}(L, X, A)-F_{1}(L, X,-A), \\
F_{1}(L, X, A):=\sqrt{L^{2}-(X+A)^{2}} .
\end{gathered}
$$

Прямыми вычислениями легко убедиться, что

$$
\begin{gathered}
F_{0}(L, X, A)=A^{2}\left(\frac{1}{F_{2}(L, X, 0, A)}+\frac{1}{F_{2}(L, X, 0,-A)}\right)+ \\
\quad+\frac{8 A^{2} X^{2}}{F_{2}(L, X, 0, A) F_{2}(L, X, 0,-A) F_{2}(L, X, A,-A)}, \\
F_{2}(L, X, A, B):=F_{1}(L, X, A)+F_{1}(L, X, B) .
\end{gathered}
$$


Отсюда немедленно заключаем, что функция $F_{0}(L, X, A)$ положительна при положительных подкоренных выражениях, входящих в ее аргументы. Также видим, что при $A \geqslant 0, X \geqslant A, X+A \leqslant L$ эта функция возрастает по $X$ и удовлетворяет оценке

$$
F_{0}(L, X, A) \geqslant A^{2}\left(\frac{1}{F_{1}(L, X,-A)}+\frac{X^{2}}{F_{1}^{3}(L, X,-A)}\right) \geqslant A^{2} \frac{L^{2}+2 A(X-A)}{\left(L^{2}-(X-A)^{2}\right)^{3 / 2}} .
$$

Всюду далее мы используем обозначение $K:=[L]$.

4.3. Случай $L>1 / 2, \alpha \leqslant 1 / 4$. В рассматриваемом случае $K \geqslant 1$, так как при $K=0$ неравенства $L>1 / 2$ и $\alpha \leqslant 1 / 4$ противоречат друг другу. Согласно соотношениям (4.2)-(4.4) имеем

$$
\begin{aligned}
& \sup _{\tau} N_{0}(L, \tau)-\inf _{\tau} N_{1}(L, \tau) \geqslant N_{0}(L, 1 / 2)-N_{1}(L, \alpha)= \\
& =\sum_{n=-K}^{K-1}\left[\frac{\sqrt{L^{2}-(n+1 / 2)^{2}}}{\varepsilon}\right]-\sum_{n=-K}^{K}\left[\frac{\sqrt{L^{2}-(n+\alpha)^{2}}}{\varepsilon}+\frac{1}{2}\right]= \\
& =\sum_{n=-K}^{K-1}\left[\frac{\sqrt{L^{2}-(n+1 / 2)^{2}}}{\varepsilon}\right]-\sum_{n=-K}^{K-1}\left[\frac{\sqrt{L^{2}-(n+\alpha)^{2}}}{\varepsilon}+\frac{1}{2}\right]= \\
& =\sum_{n=0}^{K-1}\left(2\left[\frac{\sqrt{L^{2}-(n+1 / 2)^{2}}}{\varepsilon}\right]-\left[\frac{\sqrt{L^{2}-(n+\alpha)^{2}}}{\varepsilon}+\frac{1}{2}\right]-\left[\frac{\sqrt{L^{2}-(n+1-\alpha)^{2}}}{\varepsilon}+\frac{1}{2}\right]\right),
\end{aligned}
$$

и в силу очевидной оценки $z-1 \leqslant[z] \leqslant z$ получаем

$$
\sup _{\tau} N_{0}(L, \tau)-\inf _{\tau} N_{1}(L, \tau) \geqslant \frac{S_{1}(L)}{\varepsilon}-3 K,
$$

где введены обозначения

$$
S_{1}(L):=\sum_{n=0}^{K-1} f_{1}(L, n), \quad f_{1}(L, x):=F_{0}\left(L, x+\frac{1}{2}, \frac{1}{2}-\alpha\right) .
$$

Из описанных выше свойств функции $F_{0}$ вытекает положительность величины $f_{1}(L, 0)$ и монотонное возрастание функции $f_{1}(L, x)$ по $x$. Поэтому

$$
S_{1}(L) \geqslant f_{1}(L, 0)+\int_{0}^{K-1} f_{1}(L, x) d x .
$$

При $K=1$ интеграл в правой части полученной оценки обращается в нуль, и потому

$$
S_{1}(1+\alpha) \geqslant\left.\left(2 \sqrt{(1+\alpha)^{2}-\frac{1}{4}}-2 \sqrt{\alpha}-\sqrt{1+2 \alpha}\right)\right|_{\alpha=1 / 4}>13 \varepsilon_{0} .
$$

Далее считаем, что $K \geqslant 2$. Подставляя оценку (4.5) в интеграл в (4.7) и отбрасывая слагаемое $f_{1}(L, 0)$ ввиду его положительности, получаем

$$
\begin{aligned}
& \int_{0}^{K-1} F_{0}\left(L, x+\frac{1}{2}, \frac{1}{2}-\alpha, 0\right) d x \geqslant \int_{0}^{K-1} \frac{L^{2}+(1-2 \alpha)(x+\alpha)}{16\left((K+\alpha)^{2}-(x+\alpha)^{2}\right)^{3 / 2}} d x= \\
& =\int_{\alpha}^{K-1+\alpha} \frac{(K+\alpha)^{2}+(1-2 \alpha) x}{16\left((K+\alpha)^{2}-x^{2}\right)^{3 / 2}} d x=\left.\frac{x+(1-2 \alpha)}{16 \sqrt{(K+a)^{2}-x^{2}}}\right|_{\alpha} ^{K-1+\alpha}=\frac{f_{12}(\alpha)}{16}
\end{aligned}
$$


где

$$
f_{12}(\alpha):=\frac{K-\alpha}{\sqrt{2(K+\alpha)-1}}-\frac{1-\alpha}{\sqrt{K^{2}+2 \alpha K}} .
$$

Функция $f_{12}(\alpha)$ монотонно убывает по $\alpha \in[0,1 / 4]$, так как

$$
\begin{aligned}
f_{12}^{\prime}(\alpha) & =\frac{1}{\left(K^{2}+2 \alpha K\right)^{1 / 2}}+\frac{(1-\alpha) K}{\left(K^{2}+2 \alpha K\right)^{3 / 2}}-\frac{1}{(2(K+a)-1)^{1 / 2}}-\frac{K-\alpha}{(2(K+\alpha)-1)^{3 / 2}} \leqslant \\
& \leqslant \frac{1}{K}+\frac{1}{K^{2}}-\frac{1}{(2 K-1 / 2)^{1 / 2}}-\frac{K-1 / 4}{(2 K-1 / 2)^{3 / 2}}= \\
& =\frac{1}{K}+\frac{1}{K^{2}}-\frac{3}{2^{3 / 2}(K-1 / 4)^{1 / 2}} \leqslant-\frac{3}{\sqrt{14}}+\frac{3}{4}<0 .
\end{aligned}
$$

Поэтому ввиду (4.9)

$$
\begin{aligned}
\int_{0}^{K-1} F_{0}\left(L, x, \frac{1}{2}-\alpha, 0\right) & d x \geqslant \frac{1}{16} f_{12}\left(\frac{1}{4}\right)=\frac{\sqrt{K-1 / 4}}{16 \sqrt{2}}-\frac{3}{64 \sqrt{K^{2}+K / 2}} \geqslant \\
& \geqslant\left.\left(\frac{\sqrt{K-1 / 4}}{16 \sqrt{2} \sqrt{K}}-\frac{3}{64 \sqrt{K^{2}+K / 2} \sqrt{K}}\right)\right|_{K=2} \sqrt{K} \geqslant \frac{13}{500} \sqrt{K} .
\end{aligned}
$$

Отсюда и из формул (4.6)-(4.8) выводим неравенства

$$
\begin{array}{lll}
\sup _{\tau} N_{0}(L, \tau)-\inf _{\tau} N_{1}(L, \tau)>\frac{13 \varepsilon_{0}}{\varepsilon}-3 \geqslant 10 & \text { при } \quad K=1, \\
\sup _{\tau} N_{0}(L, \tau)-\inf _{\tau} N_{1}(L, \tau) \geqslant \frac{13}{500} \frac{\sqrt{K}}{\varepsilon}-3 K & \text { при } \quad K \geqslant 2 .
\end{array}
$$

4.4. Случай $L>1 / 2,1 / 4<\alpha<1 / 2$. Как и в предыдущем случае, здесь также выполнено неравенство $K \geqslant 1$.

В силу соотношений (4.2)-(4.4) получаем

$$
\begin{aligned}
& \sup _{\tau} N_{0}(L, \tau)-\inf _{\tau} N_{1}(L, \tau) \geqslant N_{0}(L, 0)-N_{1}(L, \alpha)= \\
&=\sum_{n=-K}^{K}\left[\frac{\sqrt{L^{2}-n^{2}}}{\varepsilon}\right]-\sum_{n=-K}^{K}\left[\frac{\sqrt{L^{2}-(n+\alpha)^{2}}}{\varepsilon}+\frac{1}{2}\right]= \\
&=\sum_{n=1}^{K}\left(2\left[\frac{\sqrt{L^{2}-n^{2}}}{\varepsilon}\right]-\left[\frac{\sqrt{L^{2}-(n+\alpha)^{2}}}{\varepsilon}+\frac{1}{2}\right]-\left[\frac{\sqrt{L^{2}-(n-\alpha)^{2}}}{\varepsilon}+\frac{1}{2}\right]\right)+ \\
& \quad+\left[\frac{L}{\varepsilon}\right]-\left[\frac{\sqrt{L^{2}-\alpha^{2}}}{\varepsilon}+\frac{1}{2}\right]
\end{aligned}
$$

и потому

$$
\sup _{\tau} N_{0}(L, \tau)-\inf _{\tau} N_{1}(L, \tau) \geqslant N_{0}(L, 0)-N_{1}(L, \alpha) \geqslant \frac{S_{2}(L)}{\varepsilon}-3 K-\frac{3}{2},
$$

где

$$
S_{2}(L):=\sum_{n=0}^{K-1} f_{2}(L, n)+L-\sqrt{L^{2}-\alpha^{2}}, \quad f_{2}(L, x):=F_{0}(L, x+1, \alpha) .
$$


Функция $f_{2}(L, x)$ вновь монотонно возрастает по $x$ и положительна при $x=0$. Верны следующие оценки:

$$
\begin{gathered}
S_{2}(L) \geqslant L-\sqrt{L^{2}-\alpha^{2}}+f_{2}(L, 0)+\int_{0}^{K-1} f_{2}(L, x) d x, \\
f_{2}(L, 0)>0, \quad L-\sqrt{L^{2}-\alpha^{2}}>0 .
\end{gathered}
$$

При $K=1$ первая оценка превращается в

$$
S_{2}(1+\alpha) \geqslant\left.\left(1+\alpha-\sqrt{1+2 \alpha}+2 \sqrt{2 \alpha+\alpha^{2}}-\sqrt{4 \alpha}\right)\right|_{\alpha=1 / 4}>107 \varepsilon_{0}
$$

В случае $K \geqslant 2$ на основе (4.5) оцениваем интеграл в (4.13):

$$
\begin{aligned}
S_{2}(L) & \geqslant \int_{0}^{K-1} \frac{(K+\alpha)^{2}+2 \alpha(x+1-\alpha)}{16\left((K+\alpha)^{2}-(x+1-\alpha)^{2}\right)^{3 / 2}} d x= \\
& =\int_{1-\alpha}^{K-\alpha} \frac{(K+\alpha)^{2}+2 \alpha x}{16\left((K+\alpha)^{2}-x^{2}\right)^{3 / 2}}=\left.\frac{x+2 \alpha}{16 \sqrt{(K+a)^{2}-x^{2}}}\right|_{1-\alpha} ^{K-\alpha} \geqslant \\
& \geqslant\left.\frac{1}{16}\left(\frac{K+\alpha}{2 \sqrt{\alpha K}}-\frac{1+\alpha}{\sqrt{K^{2}+2 \alpha K+2 \alpha-1}}\right)\right|_{\alpha=1 / 2}= \\
& =\frac{1}{16}\left(\frac{K+1 / 2}{\sqrt{2 K}}-\frac{3}{2 \sqrt{K^{2}+K}}\right) \geqslant \frac{9 \sqrt{K}}{320} .
\end{aligned}
$$

Из этой оценки и формул (4.12)-(4.14) получаем неравенства

$$
\begin{array}{lcc}
\sup _{\tau} N_{0}(L, \tau)-\inf _{\tau} N_{1}(L, \tau)>\frac{107 \varepsilon_{0}}{\varepsilon}-\frac{9}{2}>102 & \text { при } \quad K=1, \\
\sup _{\tau} N_{0}(L, \tau)-\inf _{\tau} N_{1}(L, \tau) \geqslant \frac{9 \sqrt{K}}{320 \varepsilon}-3 K-\frac{3}{2} & \text { при } \quad K \geqslant 2 .
\end{array}
$$

4.5. Случай $L>1 / 2,1 / 2 \leqslant \alpha<3 / 4$. В данном случае уже следует считать, что $K \geqslant 0$.

Согласно соотношениям (4.2)-(4.4) имеем

$$
\begin{aligned}
\sup _{\tau} & N_{0}(L, \tau)-\inf _{\tau} N_{1}(L, \tau) \geqslant N_{0}(L, 0)-N_{1}(L, 1-\alpha)= \\
= & \sum_{n=-K}^{K}\left[\frac{\sqrt{L^{2}-n^{2}}}{\varepsilon}\right]-\sum_{n=-K-1}^{K}\left[\frac{\sqrt{L^{2}-(n+1-\alpha)^{2}}}{\varepsilon}+\frac{1}{2}\right]= \\
= & \sum_{n=1}^{K}\left(2\left[\frac{\sqrt{L^{2}-n^{2}}}{\varepsilon}\right]-\left[\frac{\sqrt{L^{2}-(n+1-\alpha)^{2}}}{\varepsilon}+\frac{1}{2}\right]-\right. \\
& \left.\quad-\left[\frac{\sqrt{L^{2}-(n-1+\alpha)^{2}}}{\varepsilon}+\frac{1}{2}\right]\right)+\left[\frac{L}{\varepsilon}\right]-\left[\frac{\sqrt{L^{2}-(1-\alpha)^{2}}}{\varepsilon}+\frac{1}{2}\right] .
\end{aligned}
$$

Следовательно,

$$
\sup _{\tau} N_{0}(L, \tau)-\inf _{\tau} N_{1}(L, \tau) \geqslant \frac{S_{3}(L)}{\varepsilon}-3 K-\frac{3}{2},
$$


где

$$
S_{3}(L):=\sum_{n=1}^{K} f_{3}(L, n)+L-\sqrt{L^{2}-(1-\alpha)^{2}}, \quad f_{3}(L, x):=F_{0}(L, x+1,1-\alpha) .
$$

Пусть $K=0$. Тогда сумма в определении $S_{3}$ обращается в нуль, и мы имеем

$$
S_{3}(L) \geqslant\left.\left(\alpha-\sqrt{\alpha^{2}-(1-\alpha)^{2}}\right)\right|_{\alpha=3 / 4}>8 \varepsilon_{0} .
$$

Пусть $K=1$. Тогда справедливы неравенства

$$
S_{3}(L) \geqslant\left.\left(2 \sqrt{2 \alpha+\alpha^{2}}-\sqrt{1+2 \alpha}-\sqrt{6 \alpha-3}+1+\alpha-2 \sqrt{\alpha}\right)\right|_{\alpha=3 / 4}>17 \varepsilon_{0} .
$$

Пусть теперь $K \geqslant 2$. На основе оценки (4.5) получаем

$$
\begin{aligned}
S_{3}(L) & \geqslant \int_{0}^{K-1} \frac{(K+\alpha)^{2}+2(1-\alpha)(x+\alpha)}{16\left((K+\alpha)^{2}-(x+\alpha)^{2}\right)^{3 / 2}} d x= \\
& =\int_{\alpha}^{K-1+\alpha} \frac{(K+\alpha)^{2}+2(1-\alpha) x}{16\left((K+\alpha)^{2}-x^{2}\right)^{3 / 2}} d x \geqslant \\
& \geqslant\left.\frac{1}{16}\left(\frac{K+1-\alpha}{\sqrt{2(K+\alpha)-1}}-\frac{2-\alpha}{\sqrt{K^{2}+2 \alpha K}}\right)\right|_{\alpha=3 / 4}= \\
& =\frac{\sqrt{K+1 / 4}}{16 \sqrt{2}}-\frac{5}{32 \sqrt{4 K^{2}+6 K}} \geqslant \frac{15 \sqrt{2} \varepsilon_{0}}{4} \sqrt{K} .
\end{aligned}
$$

Из формул (4.17)-(4.20) выводим неравенства

$$
\begin{aligned}
& \sup _{\tau} N_{0}(L, \tau)-\inf _{\tau} N_{1}(L, \tau)>6 \quad \text { при } K=0, K=1, \\
& \sup _{\tau} N_{0}(L, \tau)-\inf _{\tau} N_{1}(L, \tau) \geqslant \frac{15 \sqrt{2} \varepsilon_{0}}{4} \frac{\sqrt{K}}{\varepsilon}-3 K-\frac{3}{2} \quad \text { при } \quad K \geqslant 2 .
\end{aligned}
$$

4.6. Случай $L>1 / 2,3 / 4 \leqslant \alpha<1$. Здесь также следует считать, что $K \geqslant 0$. Из соотношений (4.2)-(4.4) имеем

$$
\begin{aligned}
& \sup _{\tau} N_{0}(L, \tau)-\inf _{\tau} N_{1}(L, \tau) \geqslant N_{0}(L, 1 / 2)-N_{1}(L, 1-\alpha)= \\
&= \sum_{n=-K-1}^{K}\left[\frac{\sqrt{L^{2}(n+1 / 2)^{2}}}{\varepsilon}\right]-\sum_{n=-K-1}^{K}\left[\frac{\sqrt{L^{2}-(n+\alpha)^{2}}}{\varepsilon}+\frac{1}{2}\right]= \\
&=\sum_{n=0}^{K}\left(2\left[\frac{\sqrt{L^{2}-(n+1 / 2)^{2}}}{\varepsilon}\right]-\left[\frac{\sqrt{L^{2}-(n+\alpha)^{2}}}{\varepsilon}+\frac{1}{2}\right]-\right. \\
&\left.-\left[\frac{\sqrt{L^{2}-(n+1-\alpha)^{2}}}{\varepsilon}+\frac{1}{2}\right]\right)
\end{aligned}
$$

откуда следует, что

$$
\sup _{\tau} N_{0}(L, \tau)-\inf _{\tau} N_{1}(L, \tau) \geqslant \frac{S_{4}(L)}{\varepsilon}-3 K-3
$$


где

$$
S_{4}(L):=\sum_{n=0}^{K} f_{4}(L, n), \quad f_{4}(L, x):=F_{0}\left(L, x+\frac{1}{2}, \alpha-\frac{1}{2}\right) .
$$

При $K=0$ для $S_{4}$ имеем

$$
S_{4}(\alpha) \geqslant\left.\left(2 \sqrt{\alpha^{2}-\frac{1}{4}}-\sqrt{\alpha^{2}-(1-\alpha)^{2}}\right)\right|_{\alpha=3 / 4}>83 \varepsilon_{0} .
$$

Аналогично прямыми вычислениями при $K=1$ получаем неравенство

$$
S_{4}(1+\alpha) \geqslant S_{4}(7 / 4)>126 \varepsilon_{0} .
$$

При $K \geqslant 2$ из монотонности функции $f_{4}(L, x)$ по $x$ и оценки (4.5) следует, что

$$
S_{4}(L) \geqslant f_{4}(L, 0)+\int_{0}^{K} f_{4}(L, x) d x
$$

и

$$
\begin{gathered}
\int_{0}^{K} F_{0}\left(L, x+\frac{1}{2}, \frac{1}{2}-\alpha\right) d x \geqslant \int_{0}^{K} \frac{(K+\alpha)^{2}-(2 \alpha-1)(x+1-\alpha)}{16\left((K+\alpha)^{2}-(x+1-\alpha)^{2}\right)^{3 / 2}} d x= \\
=\int_{1-\alpha}^{K+1-\alpha} \frac{L^{2}+(2 \alpha-1) x}{16\left((K+\alpha)^{2}-x\right)^{3 / 2}} d x=\left.\frac{x+(2 \alpha-1)}{16 \sqrt{(K+a)^{2}-x^{2}}}\right|_{1-\alpha} ^{K+1-\alpha} \geqslant \\
\geqslant\left.\frac{1}{16}\left(\frac{K+\alpha}{\sqrt{2 \alpha-1} \sqrt{2 K+1}}-\frac{\alpha}{\sqrt{K^{2}+2 \alpha K+2 \alpha-1}}\right)\right|_{\alpha=1}>\frac{\sqrt{K}}{25} .
\end{gathered}
$$

Отсюда и из формул (4.23)-(4.26) вытекают неравенства

$$
\begin{gathered}
\sup _{\tau} N_{0}(L, \tau)-\inf _{\tau} N_{1}(L, \tau)>80 \quad \text { при } \quad K=0, K=1, \\
\sup _{\tau} N_{0}(L, \tau)-\inf _{\tau} N_{1}(L, \tau)-1 \geqslant \frac{\sqrt{K}}{25 \varepsilon}-3 K-3 \quad \text { при } \quad K \geqslant 2 .
\end{gathered}
$$

4.7. Заключительные оценки. Из соотношений (4.10), (4.15), (4.21), (4.27) следует, что неравенство (3.4) выполнено при $K=0$ и $K=1$. Поэтому его необходимо проверить для $K \geqslant 2$. Для этого сравним правые части неравенств (4.11), (4.16), (4.22), (4.28). Так как

$$
\frac{13}{500}>\frac{15 \sqrt{2}}{4} \varepsilon_{0}, \quad \frac{9}{320}>\frac{15 \sqrt{2}}{4} \varepsilon_{0}, \quad\left(\frac{1}{25}-\frac{15 \sqrt{2}}{4} \varepsilon_{0}\right) \frac{\sqrt{K}}{\varepsilon}>4,
$$

минимальная из этих правых частей - это правая часть неравенства (4.22). Тогда достаточным условием выполнения неравенства (3.4) является оценка

$$
\frac{15 \sqrt{2} \varepsilon_{0}}{4} \frac{\sqrt{K}}{\varepsilon}-3 K-\frac{3}{2}>0 .
$$

При $K=2$ эта оценка выполнена в силу условия $(2.1)$ и, в частности, при $\varepsilon=\varepsilon_{0}$ превращается в точное равенство. Решая теперь неравенство (4.29) относительно $K$, получаем, что оно выполнено при $K \leqslant K_{\varepsilon}$, где $K_{\varepsilon}$ определено формулой (2.2). Следовательно, с учетом замкнутости спектра часть спектра оператора $\mathcal{H}_{\eta}$ вплоть до точки $\left(\left[K_{\varepsilon}\right]+1\right)^{2} / \varepsilon^{2}$ не имеет внутренних спектральных лакун при $\varepsilon \leqslant \varepsilon_{0}$, что завершает доказательство теоремы 1. 
Благодарности. Автор благодарен рецензенту за полезные замечания, которые позволили улучшить первоначальную версию статьи, и за указание на важную работу [12].

\section{Список литературы}

[1] М. М. Скриганов, А. В. Соболев, “Асимптотические оценки для спектральных зон периодических операторов Шредингера", Алгебра и анализ, 17:1 (2005), 276-288.

[2] L. Parnovski, "Bethe-Sommerfeld conjecture", Ann. Henri Poincaré, 9:3 (2008), 457-508.

[3] B. E. J. Dahlberg, E. Trubowitz, "A remark on two dimensional periodic potentials", Comment. Math. Helv., 57:1 (1982), 130-134.

[4] B. Helffer, A. Mohamed, "Asymptotics of the density of states for the Schrödinger operator with periodic electric potential", Duke Math. J., 92:1 (1998), 1-60.

[5] M. M. Skriganov, A. V. Sobolev, "Variation of the number of lattice points in large balls", Acta Arith., 120:3 (2005), 245-267.

[6] М. М. Скриганов, "Геометрические и арифметические методы в спектральной теории многомерных периодических операторов", Тр. МИАН, 171 (1985), 3-122.

[7] Y. Karpeshina, "Spectral properties of the periodic magnetic Schrödinger operator in the high-energy region. Two-dimensional case", Commun. Math. Phys., 251:3 (2004), 473-514.

[8] A. Mohamed, "Asymptotic of the density of states for the Schrödinger operator with periodic electromagnetic potential", J. Math. Phys., 38:8 (1997), 4023-4051.

[9] L. Parnovski, A. Sobolev, "On the Bethe-Sommerfeld conjecture for the polyharmonic operator", Duke Math. J., 107:2 (2001), 209-238.

[10] G. Barbatis, L. Parnovski, "Bethe-Sommerfeld conjecture for pseudo-differential perturbation", Commun. Partial Differ. Equ., 34:4 (2009), 383-418.

[11] L. Parnovski, A. V. Sobolev, "Bethe-Sommerfeld conjecture for periodic operators with strong perturbations", Invent. Math., 181:3 (2010), 467-540.

[12] C. B. E. Beeken, Periodic Schrödinger operators in dimension two: constant magnetic fields and boundary value problems, PhD thesis, University of Sussex, Brighton, 2002.

[13] D. Borisov, G. Cardone, T. Durante, "Homogenization and uniform resolvent convergence for elliptic operators in a strip perforated along a curve", Proc. Roy. Soc. Edinburgh Sect. A, 146:6 (2016), 1115-1158.

[14] D. Borisov, G. Cardone, L. Faella, C. Perugia, "Uniform resolvent convergence for a strip with fast oscillating boundary", J. Differ. Equ., 255:12 (2013), 4378-4402.

[15] D. Borisov, R. Bunoiu, G. Cardone, "Waveguide with non-periodically alternating Dirichlet and Robin conditions: homogenization and asymptotics", Z. Angew. Math. Phys., 64:3 (2013), 439-472.

[16] D. Borisov, R. Bunoiu, G. Cardone, "On a waveguide with frequently alternating boundary conditions: homogenized Neumann condition", Ann. Henri Poincaré, 11:8 (2010), $1591-1627$.

[17] D. Borisov, G. Cardone, "Homogenization of the planar waveguide with frequently alternating boundary conditions", J. Phys. A.: Math. Gen., 42:36 (2009), 365205, 21 pp.

[18] D. Borisov, G. Cardone, T. Durante, "Norm-resolvent convergence for elliptic operators in domain with perforation along curve", C. R. Math. Acad. Sci. Paris, 352:9 (2014), 679-683.

[19] D. Borisov, R. Bunoiu, G. Cardone, "Homogenization and asymptotics for a waveguide with an infinite number of closely located small windows", Пробл. матем. анализа, 58:6 (2011), 59-68. 